\title{
Deadlines in stochastic contests
}

Citation for published version (APA):

Lang, M., Seel, C., \& Strack, P. (2014). Deadlines in stochastic contests. Journal of Mathematical Economics, 52, 134-142. https://doi.org/10.1016/j.jmateco.2013.10.003

Document status and date:

Published: 01/05/2014

DOI:

10.1016/j.jmateco.2013.10.003

Document Version:

Publisher's PDF, also known as Version of record

Document license:

Taverne

Please check the document version of this publication:

- A submitted manuscript is the version of the article upon submission and before peer-review. There can be important differences between the submitted version and the official published version of record.

People interested in the research are advised to contact the author for the final version of the publication, or visit the DOI to the publisher's website.

- The final author version and the galley proof are versions of the publication after peer review.

- The final published version features the final layout of the paper including the volume, issue and page numbers.

Link to publication

\footnotetext{
General rights rights.

- You may freely distribute the URL identifying the publication in the public portal. please follow below link for the End User Agreement:

www.umlib.nl/taverne-license

Take down policy

If you believe that this document breaches copyright please contact us at:

repository@maastrichtuniversity.nl

providing details and we will investigate your claim.
}

Copyright and moral rights for the publications made accessible in the public portal are retained by the authors and/or other copyright owners and it is a condition of accessing publications that users recognise and abide by the legal requirements associated with these

- Users may download and print one copy of any publication from the public portal for the purpose of private study or research.

- You may not further distribute the material or use it for any profit-making activity or commercial gain

If the publication is distributed under the terms of Article $25 \mathrm{fa}$ of the Dutch Copyright Act, indicated by the "Taverne" license above, 


\title{
Deadlines in stochastic contests
}

\author{
Matthias Lang ${ }^{\mathrm{a}}$, Christian Seel ${ }^{\mathrm{b}, *}$, Philipp Strack $^{\mathrm{c}}$ \\ a Department of Economics, Humboldt-Universität zu Berlin, Germany \\ ${ }^{\mathrm{b}}$ Department of Economics, Maastricht University, The Netherlands \\ ${ }^{\mathrm{c}}$ Microsoft Research, United States
}

\section{A R T I C L E I N F O}

\section{Article history:}

Received 3 May 2013

Received in revised form

20 August 2013

Accepted 7 October 2013

Available online 17 October 2013

\section{Keywords:}

Contest

All-pay auction

Research tournament

\begin{abstract}
A B S T R A C T
We consider a two-player contest model in which breakthroughs arrive according to privately observed Poisson processes. Each player's process continues as long as she exerts costly effort. The player who collects the most breakthroughs until a predetermined deadline wins a prize.

We derive Nash equilibria of the game depending on the deadline. For short deadlines, there is a unique equilibrium in which players use identical cutoff strategies, i.e., they continue until they have a certain number of successes. If the deadline is long enough, the symmetric equilibrium distribution of an all-pay auction is an equilibrium distribution over successes in the contest. Expected efforts may be maximal for a short or intermediate deadline.
\end{abstract}

(C) 2013 Elsevier B.V. All rights reserved.

\section{Introduction}

There is a large amount of literature on all-pay auctions that are often informally motivated as reduced-form models of a contest. ${ }^{1}$ The bid in the auction serves as a proxy for the effort or production cost each participant incurs in the contest; think, for example, of an R\&D, job promotion, or procurement contest. This paper formally raises the question how all-pay auctions relate to stochastic contests.

For this purpose, we consider a contest with two main distinctive features. First, each player observes her own progress over time, but has no information about the progress of her rivals. Contrary to a silent timing game, however, each player's progress is stochastic. Second, the contest success function has a cumulative structure, i.e., separate steps add up to the final success.

Many procurement and R\&D contests feature these properties: typically contestants conduct their research in secrecy and there are several steps in the development of a jet fighter or a vaccine, for example. But the model also accommodates other contests such as grant competitions: every contestant writes a grant application

\footnotetext{
* Correspondence to: Maastricht University, School of Business and Economics, 6211 LM, Maastricht, The Netherlands. Tel.: +31 0433883651.

E-mail addresses: lang@uni-bonn.de (M. Lang), c.seel@maastrichtuniversity.nl (C. Seel), philipp.strack@gmail.com (P. Strack).

1 For example, Hillman and Samet (1987), Hillman and Riley (1989), Baye et al. (1996), Che and Gale (1998), Siegel (2009), and Bos (2012) study all-pay auctions with a continuous bid space, while Dechenaux et al. $(2003,2006)$, and Cohen and Sela (2007) scrutinize all-pay auctions in which the set of bids is countable.
}

without knowing the quality of the other applications. Spending more time on the application might increase its quality, and thereby the chance of receiving the grant.

Although most real-world contests and theoretical definitions of a contest contain a deadline, most of the literature abstracts from the deadline by either analyzing static models or setting the deadline to infinity. This paper explicitly focuses on the influence of the deadline on the Nash equilibria of the contest. More precisely, we identify equilibria of the contest for different deadlines and compare them.

Formally, we consider a stochastic contest model in which players decide when to stop privately observed Poisson processes. As long as a player does not stop his process, successes arrive according to a Poisson process. The player who accumulates most Poisson arrivals until the contest deadline wins a prize. Ties are broken randomly. Players maximize expected profits.

The analysis proceeds as follows. First, we recall the bidding distribution in a Nash equilibrium of an all-pay auction in which the set of bids is countable as a benchmark. Under a genericity assumption, we show in Proposition 1 that this distribution is the unique symmetric equilibrium distribution of the auction.

A stopping strategy in the contest induces a probability distribution over the number of successes at the stopping time. Proposition 2 derives a time bound above which the symmetric Nash equilibrium distribution of a discrete all-pay auction is an equilibrium distribution of the contest. By Proposition 3, the equilibrium set of the all-pay auction is identical to the equilibrium set of the contest for an infinite deadline. Hence, the all-pay auction provides a suitable model of a contest which lasts sufficiently long. 
Table 1

Summary of our equilibrium characterization

\begin{tabular}{llll}
\hline Deadline & Equilibrium type & Characterization & Binding deadline \\
\hline Short & Cutoff & Unique equilibrium & Yes \\
Intermediate & $\begin{array}{l}\text { Time-dependent } \\
\text { cutoff }\end{array}$ & Example & Yes \\
& All-pay auction & An equilibrium & No \\
Long & All-pay auction & Equilibrium & No \\
& & equivalence & \\
\hline
\end{tabular}

For short and intermediate deadlines, it is impossible to replicate the equilibrium distribution of the all-pay auction in the contest. For short deadlines, Propositions 4 and 5 uniquely characterize equilibrium strategies as cutoff strategies: players only stop if they reach a certain number of successes or the game ends. Intuitively, they strictly prefer to continue for all low values. Yet, they strictly prefer to stop above some cutoff value, since the probability that the rival reaches this value until the deadline is too low to make continuation profitable.

Proposition 6 shows that - depending on the parameters - there might be an intermediate region for which neither a cutoff equilibrium, nor an all-pay auction equilibrium exists. In this case, we obtain equilibria in which strategies depend both on the remaining time and the number of successes. We discuss the properties of such an equilibrium in an example.

We define a deadline to be binding for an equilibrium if a player could increase her payoff by unilaterally postponing the deadline. Proposition 7 shows that the deadline is non-binding in the allpay auction type equilibrium and binding in any other equilibrium. Intuitively, only in the all-pay auction equilibrium, each player is indifferent whether to stop at any point of her support at any point in time. Thus, no player would benefit from a longer deadline.

Table 1 briefly summarizes our results for different deadlines.

Finally, we compare expected equilibrium efforts for different deadlines. We discuss an example to show that the equilibrium effort for a short deadline could be higher than for a longer deadline. Intuitively, a shorter deadline reduces the possible competition time, but might increase the intensity of competition in the remaining time. Hence, a contest designer who wants to maximize equilibrium efforts is sometimes better off with a short deadline. In this case, the equilibrium of the contest differs from that of an all-pay auction which illustrates a potential drawback of analyzing all-pay models as a shortcut for contests.

In the literature, there are surprisingly few multi-period contest models in which each player's decision problem is dynamic. The most prominent model in this class in which there is no interaction between the players over time is Taylor (1995). He analyzes a $T$ period model. The technology in Taylor (1995) is quite different from this paper: in each period, each player decides whether she wants to take an additional draw. A draw is a realization of a random variable. The distribution of the random variable is commonly known and identical across players and time periods. At the deadline $T$, the player with the highest overall draw wins. In equilibrium, each player stops only if he has a draw above a certain cutoff. Equilibrium efforts are monotonically increasing in the deadline.

Seel and Strack (2013) consider a contest model with the same information structure as in the present paper without a deadline. In their framework, players have to stop a Brownian motion with a drift. They bear no costs for a later stopping time, but they have to stop if their process hits zero. In equilibrium, players do not stop immediately even if the drift is negative.

We proceed as follows. In Section 2, we set up the model. Section 3 contains the main results of the paper. Section 4 discusses the results and concludes. Proofs not provided in the main text are relegated to the Appendix.

\section{The contest model}

Consider a contest with a fixed deadline $T<\infty$ and two players. At every point $t \leq T$, each player $i \in\{1,2\}$ privately observes a time-homogeneous Poisson process $X_{t}^{i}$ with intensity $\lambda \in \mathbb{R}_{+}$and jump size 1 . A strategy of player $i$ is a stopping time $\tau^{i} \leq T$ with respect to the natural filtration $\mathcal{F}_{t}^{i}$ generated by the process $X_{t}^{i}$. The stopping time induces a probability distribution over values of the process at the stopping point. We denote this distribution by $F^{i}: \mathbb{N}_{0} \rightarrow[0,1]$, where $F^{i}(x)=\mathbb{P}\left(X_{\tau^{i}}^{i} \leq x\right)$. The associated probability measure is denoted by $f^{i}(x)$. Stopping at time $t$ entails costs of $c t$.

To interpret the stopping decision, think about a player $i$ who chooses an effort level $\eta_{t}^{i} \in\{0,1\}$ at any time $t \leq T$. The effort decision $\eta_{t}^{i}=1$ indicates continuing to work on a project at a flow cost of effort of $c$, while $\eta_{t}^{i}=0$ indicates not working on the project anymore. In this model, the arrival rate of the stochastic process $X_{t}^{i}$ at time $t$ equals $\lambda \eta_{t}^{i}$. This formulation of the problem in terms of efforts is mathematically equivalent to our formulation in terms of stopping times as $\int \eta_{t}^{i} \mathrm{~d} t$ equals the realization of the stopping time $\tau^{i}$. Hence, we sometimes refer to the stopping time $\tau^{i}$ as a player's effort.

The player who has more Poisson arrivals at her stopping time $\tau^{i}$ wins a prize $p$. Ties are broken randomly. Thus, each player's payoff is

$\pi^{i}= \begin{cases}p-c \tau^{i} & \text { if } X_{\tau^{i}}^{i}>X_{\tau^{j}}^{j} \\ \frac{p}{2}-c \tau^{i} & \text { if } X_{\tau^{i}}^{i}=X_{\tau^{j}}^{j} \\ -c \tau^{i} & \text { otherwise. }\end{cases}$

Players maximize expected payoffs. Define the payoff process $\left(\Pi_{t}^{i}\right)_{t \in \mathbb{R}_{+}}$of player $i$ as his expected payoff of stopping immediately, i.e.,

$$
\begin{aligned}
\Pi_{t}^{i} & =\mathbb{E}\left(\pi^{i} \mid X_{t}^{i}, \tau^{i}=t\right) \\
& =p \mathbb{P}\left(X_{t}^{i}>X_{\tau^{j}}^{j}\right)+\frac{p}{2} \mathbb{P}\left(X_{t}^{i}=X_{\tau^{j}}^{j}\right)-c t .
\end{aligned}
$$

\section{Equilibrium analysis}

In this section, we derive Nash equilibria depending on the deadline. We use Nash equilibrium as the solution concept since no player receives any information about his rival over time.

\subsection{Long deadlines}

As a benchmark, we first consider the Nash equilibrium of a related static model, the all-pay auction with discrete bids. We then show that the symmetric equilibrium distribution of the allpay auction is also an equilibrium distribution in the contest and construct an explicit stopping time which leads to this distribution.

\subsubsection{The all-pay auction}

Consider a model with two risk-neutral players indexed by $i=1,2$. A pure strategy of player $i$ is a bid $x^{i} \in \mathbb{N}_{0}$. A mixed strategy of player $i$ is a probability measure $f^{i}: \mathbb{N}_{0} \mapsto[0,1]$. Denote the associated cumulative distribution function by $F^{i}(z)=\mathbb{P}\left(x^{i} \leq z\right)$ $=\sum_{y=0}^{z} f^{i}(y)$. The agent with the highest bid wins a prize $\hat{p} \in \mathbb{R}_{+}$ and both players pay their bids $x^{i}$. Ties are broken randomly. Hence, the profit of player $i$ from bidding $x^{i}$ is

$u^{i}\left(x^{i}, x^{j}\right)= \begin{cases}\hat{p}-x^{i} & \text { if } x^{i}>x^{j} \\ \frac{\hat{p}}{2}-x^{i} & \text { if } x^{i}=x^{j} \\ -x^{i} & \text { otherwise. }\end{cases}$ 
To get a unique symmetric equilibrium, we impose the following genericity assumption:

Assumption 1. $\frac{\hat{p}}{2} \notin \mathbb{N}$.

Assumption 1 rules out non-generic parameter settings in which the prize is an even integer. In those cases, infinitely many equilibria exist; see Baye et al. (1994) and Cohen and Sela (2007). By standard arguments in the literature on all-pay auctions, players have to be indifferent in any symmetric Nash equilibrium between all bids $x \in\{0,1, \ldots, \bar{x}\}$, where $\bar{x}=\max \{x: f(x)>0\}$. Hence, we obtain the difference equation

$\frac{f(x)+f(x+1)}{2}=\frac{1}{\hat{p}} \quad \forall x<\bar{x}$

subject to $\sum_{0}^{\bar{x}} f(x)=1$ and $f(x) \geq 0$ for all $x$. Given Assumption 1 , it is straightforward to see that the difference equation has a unique solution. Therefore, the proof of the following proposition is omitted.

Proposition 1. Suppose Assumption 1 holds. Then the unique symmetric Nash equilibrium of the all-pay auction is

$$
\begin{aligned}
f^{1}(x) & =f^{2}(x)=f(x) \\
& = \begin{cases}\frac{2}{\hat{p}}\left(\frac{\hat{p}-\bar{x}}{2}\right) & \text { if } x \leq \bar{x} \text { and } x \text { is even } \\
\frac{2}{\hat{p}}\left(1-\frac{\hat{p}-\bar{x}}{2}\right) & \text { if } x<\bar{x} \text { and } x \text { is odd } \\
0 & \text { otherwise, }\end{cases}
\end{aligned}
$$

where $\bar{x}=\max \left\{x \in \mathbb{N}_{0}: x\right.$ even and $\left.x<\hat{p}\right\}$.

The expected profit of a player equals her expected profit of bidding 0 , since bidding 0 is a best response to the equilibrium strategy of the opponent. Hence, the expected profit is $\frac{\hat{p}}{2} f(0)=\frac{\hat{p}-\bar{x}}{2}$.

The probability measure in Eq. (3) serves as a benchmark for the contest model. In particular, note that $f(x)>0$ if and only if $x \leq \bar{x}$.

\subsubsection{The contest with a long deadline}

In this subsection, we relate the symmetric equilibrium distribution of the all-pay auction to that of the contest. In a first step, we derive a result for distributions with "connected support". More precisely, we show that there exists a bounded stopping time which induces such a distribution if the contest length is above a threshold value. For this purpose, define $\tilde{x}=\max \{x: f(x)>0\}$.

Lemma 1. Consider an arbitrary distribution $F(x)$ on $\mathbb{N}_{0}$ such that $f(x)>0$ if and only if $x \leq \tilde{x}$. Additionally, suppose $T \geq-\frac{1}{\lambda}$ $\sum_{x=0}^{\tilde{x}-1} \log \left(\frac{f(x)}{1-F(x-1)}\right)$. Then there exists a strategy $\tau$ such that $\mathbb{P}\left(X_{\tau} \leq\right.$ $x)=F(x)$.

Intuitively, the strategy which we construct in the proof of Lemma 1 is simple: a player stops only if no success arrives within a certain time interval, which depends on the value of the process. Hence, the strategy conditions only on the value of the process and the time spent at this value.

In the next step, we show that the all-pay auction equilibrium distribution is an equilibrium distribution of the contest. For this purpose, we define $\mathcal{L} \Pi^{i}: \mathbb{N}_{0} \rightarrow \mathbb{R}$ as the expected infinitesimal change of the payoff process $\Pi_{t}^{i}$ defined in Eq. (1) at any point $X_{t}^{i}=x$ by

$$
\begin{aligned}
\left(\mathcal{L} \Pi^{i}\right)(x) & =\lim _{\Delta \searrow 0} \frac{1}{\Delta} \mathbb{E}\left(\Pi_{t+\Delta}^{i}-\Pi_{t}^{i} \mid X_{t}^{i}=x\right) \\
& =\lim _{\Delta \searrow 0} \frac{p}{\Delta} \mathbb{E}\left(F^{j}\left(X_{t+\Delta}^{i}\right)-\frac{1}{2} f^{j}\left(X_{t+\Delta}^{i}\right)\right.
\end{aligned}
$$

$$
\begin{aligned}
& \left.-F^{j}\left(X_{t}^{i}\right)+\frac{1}{2} f^{j}\left(X_{t}^{i}\right) \mid X_{t}^{i}=x\right)-c \\
= & \frac{p \lambda}{2}\left(f^{j}(x)+f^{j}(x+1)\right)-c \\
= & c\left(\hat{p} \frac{f^{j}(x)+f^{j}(x+1)}{2}-1\right) .
\end{aligned}
$$

In the proof of the following proposition, we adapt the indifference Eq. (2) for the all-pay auction to an indifference equation for the process. Hence, we set the infinitesimal change in expected payoff $\mathcal{L} \Pi^{i}: \mathbb{N}_{0} \rightarrow \mathbb{R}$ equal to zero.

Proposition 2. Denote the probability measure defined in (3) by $f$. Set $\hat{p}=\frac{\lambda p}{c}$ and let

$T \geq \frac{1}{\lambda} \sum_{x=0}^{\bar{x} / 2-1} \log \left(\frac{(\hat{p}-2 x)(\bar{x}-2 x)}{(\hat{p}-\bar{x})(2+\bar{x}-\hat{p})}\right)$.

Then there exists a Nash equilibrium of the contest which induces the probability measure $f$ for each player.

Proof. The probability measure of the symmetric Nash equilibrium of the all-pay auction defined in Eq. (3) has the property $f(x)>0$ if and only if $x \leq \bar{x}$. Thus, by Lemma 1 , there exists a strategy which induces it if

$$
\begin{aligned}
T & \geq-\frac{1}{\lambda} \sum_{x=0}^{\bar{x}-1} \log \left(\frac{f(x)}{1-F(x-1)}\right) \\
& =-\frac{1}{\lambda} \sum_{x=0}^{\bar{x} / 2-1}\left(\log \left(\frac{f(2 x)}{1-F(2 x-1)}\right)+\log \left(\frac{f(2 x+1)}{1-F(2 x)}\right)\right) \\
& =-\frac{1}{\lambda} \sum_{x=0}^{\bar{x} / 2-1}\left(\log \left(\frac{\hat{p}-\bar{x}}{\hat{p}-2 x}\right)+\log \left(\frac{2-\hat{p}+\bar{x}}{\hat{p}-(2 x+\hat{p}-\bar{x})}\right)\right) \\
& =\frac{1}{\lambda} \sum_{x=0}^{\bar{x} / 2-1} \log \left(\frac{(\hat{p}-2 x)(\bar{x}-2 x)}{(\hat{p}-\bar{x})(2+\bar{x}-\hat{p})}\right) .
\end{aligned}
$$

To show that such a strategy is an equilibrium strategy in the contest, it remains to verify that no player has a profitable deviation. Consider the expression for the infinitesimal change in expected payoffs in Eq. (4) for $\hat{p}=\frac{p \lambda}{c}$. By the indifference argument in Proposition 1 , for all $x<\bar{x}$,

$\hat{p} \frac{f^{j}(x)+f^{j}(x+1)}{2}-1=0$.

Therefore, $\left(\mathcal{L} \Pi^{i}\right)(x)=0$ for all $x<\bar{x}$. By the same argument, for all points $x \geq \bar{x},\left(\mathcal{L} \Pi^{i}\right)(x) \leq p \lambda \frac{f^{j}(\bar{x})}{2}-c<0$. Hence, $\left(\mathcal{L} \Pi^{i}\right)(x) \leq 0$ for all $x$, i.e., the process $\Pi_{t}^{i}$ is a supermartingale and a martingale until the process $X^{i}$ reaches $\bar{x}$.

Denote by $\tau$ a stopping time inducing the equilibrium distribution $F$ and by $\tau_{0}$ the strategy which stops immediately. By Doob's optional stopping theorem (Rogers and Williams, 2000, p. 189), for any bounded stopping time $\hat{\tau}, \mathbb{E}\left(\Pi_{\hat{\tau}}\right) \leq \mathbb{E}\left(\Pi_{\tau_{0}}\right)=\mathbb{E}\left(\Pi_{\tau}\right)$. Thus, there is no profitable deviation.

Therefore, to find an equilibrium distribution in a contest with a long deadline, it suffices to calculate $\frac{\lambda p}{c}$ and insert it for $\hat{p}$ in the all-pay auction equilibrium.

We now briefly discuss the case of an infinite deadline $T=\infty$. As we want to use the same definition of strategies and payoffs as before, we restrict attention to stopping times that satisfy $\tau<\infty$ almost surely.

Proposition 3. For $T=\infty$, the set of equilibrium distributions of the contest equals the set of equilibrium distributions of the all-pay auction. 
The idea of the proof is similar to the proofs of Lemma 1 and Proposition 2. We first show that the strategy from the proof of Lemma 1 can be used to induce any distribution. Then, we show that the expected costs of inducing a particular distribution equal the costs in the all-pay auction. Hence, the contest with deadline $T=\infty$ and the all-pay auction are strategically equivalent. Therefore, they also have the same set of Nash equilibrium distributions.

\subsection{Short deadlines}

In this section, we construct the unique equilibrium for short deadlines. To do so, we first introduce cutoff strategies, i.e., strategies that stop only if the process reaches a certain cutoff.

Definition 1. The cutoff strategy $\tau_{\bar{x}}$ with cutoff $\bar{x} \in \mathbb{N}_{0}$ is the stopping time

$\tau_{\bar{x}}=\inf \left\{t \geq 0: X_{t} \geq \bar{x}\right\}$

As the arrival rate of successes is Poisson distributed, the probability measure induced by a cutoff strategy until the deadline $T$ satisfies

$f(x)=\mathbb{P}\left(X_{\tau}=x\right)= \begin{cases}\frac{e^{-\lambda T}}{x !}(\lambda T)^{x} & \text { if } x<\bar{x} \\ 1-\sum_{x=0}^{\bar{x}-1} \frac{e^{-\lambda T}}{x !}(\lambda T)^{x} & \text { if } x=\bar{x} \\ 0 & \text { otherwise. }\end{cases}$

The probability measure of a cutoff strategy enables us to derive conditions for the existence of equilibria in which both players use the same cutoff strategy. We begin with cutoffs 0 and 1 .

Proposition 4. If $c>\frac{1}{2} \lambda p$, both players stop immediately in the unique equilibrium. If $c<\frac{1}{2} \lambda p$ and $T<-\frac{1}{\lambda} \log \left(1-\frac{2 c}{\lambda p}\right)$, both players use a cutoff strategy with cutoff 1 in the unique equilibrium.

Proof. If $c>\frac{p \lambda}{2}$, the expectation of the infinitesimal generator of the payoff process satisfies

$\left(\mathscr{L} \Pi^{i}\right)(x)=p \lambda \frac{f^{j}(x)+f^{j}(x+1)}{2}-c \leq \frac{p \lambda}{2}-c<0$

i.e., it is optimal to stop immediately for each player independently of the distribution of the other player. Put differently, in equilibrium, each player chooses a cutoff of 0 .

Let us now consider the case $c<\frac{p \lambda}{2}$. We have $f^{j}(0) \geq e^{-\lambda T}$, because $e^{-\lambda T}$ is the value for $f^{j}(0)$ if a player never stops at zero before the deadline $T$. Thus, $f^{j}(x)+f^{j}(x+1) \leq 1-e^{-\lambda T}$ for all $x \geq 1$. Hence, continuation at $x \geq 1$ is unprofitable if

$\left(\mathcal{L} \Pi^{i}\right)(x) \leq p \lambda \frac{1-e^{-\lambda T}}{2}-c<0$,

i.e., $T<-\frac{1}{\lambda} \log \left(1-\frac{2 c}{\lambda p}\right)$. Since each player stops at 1 in any equilibrium for $T<-\frac{1}{\lambda} \log \left(1-\frac{2 c}{\lambda p}\right)$, we obtain $f^{i}(0)+f^{i}(1)=$ $f^{j}(0)+f^{j}(1)=1$. Therefore, each player strictly prefers not to stop at zero if $c<\frac{p \lambda}{2}$. Hence, for these parameters, there is a unique equilibrium with cutoff 1 for each player.

An equilibrium with cutoff 0 exists if costs are high. In this case, no player would ever have an incentive to continue. An equilibrium with cutoff 1 for each player exists if costs are not too high and the duration of the contest is very short.

If $c=\frac{1}{2} \lambda p$, each player stops in any equilibrium if $X_{t} \geq 1$. Hence, each player only places positive probability mass on 0 and 1. In this case, the gain in winning probability from stopping at
1 compared to stopping at 0 is $\frac{f(0)+f(1)}{2}=\frac{1}{2}$ for any distribution of the probability mass of the other player between 0 and 1 . For $c=\frac{1}{2} \lambda p$, the expected gain in winning probability at $X_{t}=0$ is exactly offset by the expected costs, i.e., any strategy at $X_{t}=0$ is optimal. The intuition is the same as for the all-pay auction with $\hat{p}=2$ : no player bids above 1 in equilibrium, but a continuum of equilibria with different randomizations of bids between 0 and 1 exists.

We now characterize conditions for the existence of cutoff equilibria with cutoffs above 1 .

Lemma 2. Identical cutoff strategies with cutoff $\bar{x} \geq 2$ are the unique Nash equilibrium if the following inequalities are satisfied:

$$
\begin{aligned}
& e^{-\lambda T}\left(\frac{1}{x !}(\lambda T)^{x}+\frac{1}{(x+1) !}(\lambda T)^{x+1}\right)>\frac{2 c}{\lambda p} \text { for } x \in\{0, \bar{x}-2\} \\
& 1-e^{-\lambda T} \sum_{x=0}^{\bar{x}-2} \frac{1}{x !}(\lambda T)^{x}>\frac{2 c}{\lambda p} \\
& 1-e^{-\lambda T} \sum_{x=0}^{\bar{x}-1} \frac{1}{x !}(\lambda T)^{x}<\frac{2 c}{\lambda p} .
\end{aligned}
$$

Intuitively, the inequalities (6) ensure that each player prefers to continue for any point below $\bar{x}-1$; inequality (7) ensures that continuation is profitable at $\bar{x}-1$. Inequality (8) states that continuation at $\bar{x}$ is unprofitable.

In the following, we reduce the possible candidates for $\bar{x}$ to a singleton. For this purpose, define

$\hat{x}=\min \left\{x \in \mathbb{N}_{0}: 1-e^{-\lambda T} \sum_{y=0}^{x-1} \frac{1}{y !}(\lambda T)^{y} \leq \frac{2 c}{\lambda p}\right\}$.

Notice that $\hat{x}$ is well-defined and unique, because $\frac{1}{y !}(\lambda T)^{y}>0$ for all $y$. To avoid a multiplicity of equilibria, we rule out non-generic parameter settings in which inequality (8) holds with equality by imposing a condition similar to Assumption 1:

\section{Assumption 2.}

$1-\sum_{y=0}^{\hat{x}-1} \frac{e^{-\lambda T}}{y !}(\lambda T)^{y} \neq \frac{2 c}{\lambda p}$.

If this assumption is violated, players could be indifferent between stopping and continuing at $\hat{x}$ which leads to a continuum of equilibria as we have discussed after Proposition 4. Given Assumption 2, we show that there is a unique equilibrium for short deadlines. The next lemma provides the unique candidate for $\bar{x}$ and a simple condition for the existence of a cutoff equilibrium.

Lemma 3. Suppose Assumption 2 holds. Then in any equilibrium in identical cutoff strategies, we have $\bar{x}=\hat{x}$. To verify the conditions of Lemma 2, it suffices to verify

$1+\lambda T>\frac{2 c}{\lambda p} e^{\lambda T}$

$\frac{1}{(\hat{x}-2) !}(\lambda T)^{\hat{x}-2}+\frac{1}{(\hat{x}-1) !}(\lambda T)^{\hat{x}-1}>\frac{2 c}{\lambda p} e^{\lambda T}$.

Thus, we are left with the inequalities from (6) with $\bar{x}=\hat{x}$. From our definition of $\hat{x}$, we can deduce that inequalities (7) and (8) are automatically satisfied. 
Solving inequality (10) for $T$ in the case $c<\frac{1}{2} \lambda p$, we obtain

$T<-\frac{1}{\lambda}\left(1+W_{-1}\left(-\frac{2 c}{p \lambda e}\right)\right)$,

where $W_{-1}:\left[-\frac{1}{e}, 0\right) \rightarrow(-\infty,-1]$ is the lower branch of the Lambert $W$-function implicitly defined by $x=W_{-1}(x) e^{W_{-1}(x)}$. Combining this result with Proposition 4 , we can explicitly define the time bound $\underline{T}$ :

$\underline{T}(z)= \begin{cases}\infty & \text { for } z>\frac{1}{2} \\ -\frac{1}{\lambda} \min \left\{\log (1-2 z), W_{-1}\left(-2 \frac{z}{e}\right)+1\right\} & \text { for } z<\frac{1}{2} .\end{cases}$

The next proposition shows that this time bound is necessary for the existence of a cutoff equilibrium.

Proposition 5. If $T>\underline{T}\left(\frac{c}{\lambda p}\right)$, there exists no equilibrium in cutoff strategies. Now, suppose Assumption 2 holds. If $T<\underline{T}\left(\frac{c}{\lambda p}\right)$ and either condition (11) or one of the conditions in Proposition 4 is satisfied, there is a unique equilibrium. In equilibrium, both players use symmetric cutoff strategies with cutoff $\hat{x}$ defined in Eq. (9).

Proof. We prove the first statement by contradiction. Suppose there is a cutoff equilibrium with cutoff $\bar{x} \geq 2$ and

$e^{-\lambda T}(1+\lambda T)<\frac{2 c}{\lambda p}$

Note that the left-hand side of Eq. (13) equals the probability mass on 0 and 1 in every cutoff equilibrium with cutoff $\bar{x} \geq 2$ and deadline $T$. The expectation of the infinitesimal generator of the payoff process at 0 is

$$
\begin{aligned}
\left(\mathscr{L} \Pi^{i}\right)(0) & =p \lambda\left(\frac{f^{j}(0)+f^{j}(1)}{2}\right)-c \\
& =p \lambda e^{-\lambda T}\left(\frac{1+\lambda T}{2}\right)-c<0 .
\end{aligned}
$$

In the remainder of this proof, we show that a negative expectation of the infinitesimal generator at 0 implies that there is an $\epsilon$-neighborhood of $T$ where the player prefers to stop at $X_{t}=0$. Intuitively, in this case the loss from continuation at 0 is higher than the potential gain from continuation at 1 .

Denote the expected additional gain from optimal continuation of a player at time $t$ at value 1 compared to stopping by $v(1, t)$. Note that

$0 \leq v(1, t) \leq p\left(1-e^{-\lambda(T-t)}\right)$,

since $1-e^{-\lambda(T-t)}$ is the probability of at least one additional success until time $T$ multiplied by 1 , the maximal increase in winning probability. However,

$\lim _{t \rightarrow T} p\left(1-e^{-\lambda(T-t)}\right)=0$.

The potential gain from continuation at time $t$ at value 1 is arbitrarily close to 0 as $t$ approaches $T$ by Eq. (15) and continuity of $v(1, t)$. Moreover, the expected direct gain from continuation is negative by Eq. (14). Hence, there exists an $\epsilon>0$ such that it is strictly optimal to stop at value 0 for $t \in(T-\epsilon, T)$. This contradicts optimality of continuation at 0 in a cutoff equilibrium with cutoff $\bar{x} \geq 2$.

The second statement follows directly from Lemmas 2 and 3 in combination with Proposition 4.

To sum up, we have provided an equilibrium characterization for cutoff equilibria. Given the parameters, it is simple to verify whether the conditions of Proposition 4 or 5 are met, i.e., whether a cutoff equilibrium exists.

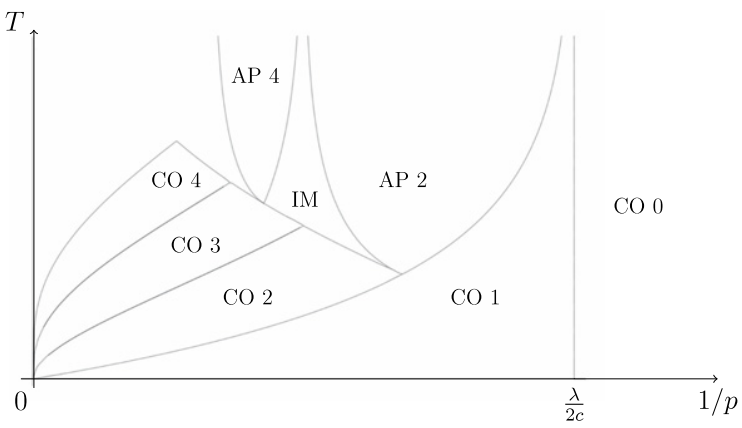

Fig. 1. Equilibrium structure. $\mathrm{CO} x$ denotes a region in which the unique equilibrium is an equilibrium with cutoff $x, \mathrm{AP} x$ denotes a region in which an all-pay auction equilibrium with $\bar{x}=x$ exists and IM denotes an intermediate region in which none of these equilibria exists.

\subsection{Intermediate deadlines}

So far, we have considered the two extreme cases, either the deadline is short enough that both players use cutoff strategies in equilibrium or it is sufficiently long such that the equilibrium distribution of an all-pay auction can be induced. Depending on the parameters, there might be a third, intermediate region:

Proposition 6. For some values $\lambda, c, p$, and $T$, there exists neither a cutoff equilibrium nor an all-pay auction equilibrium, but a different type of equilibrium exists.

In the proof of Proposition 6, we first provide an example of a parameter region for which none of the above types of equilibria exists. In a second step, we derive an equilibrium for parameters in this region. In this equilibrium, each player stops at value $X_{t}=0$ at a certain time $0<t_{0}<T$, always continues at $X_{t}=1$ and always stops at $X_{t}=2$. Intuitively, continuation from 0 to 1 has a negative expectation. Before time $t_{0}$, this effect is offset by the potential gain of continuation from 1 to 2 . After time $t_{0}$, the probability to reach value 2 is too small to make continuation at 0 profitable. Hence, continuation at 0 is only profitable if the remaining time to the deadline is long enough. After some time without a breakthrough, players become disappointed and stop their process.

In contrast to the cutoff equilibria, in the intermediate region the stopping decision depends on the value $X_{t}$ and the time $t$. Moreover, in contrast to the all-pay auction equilibria for long deadlines, the equilibrium distribution for intermediate deadlines depends on the deadline $T$.

\section{Discussion}

The equilibrium construction has characterized three regions of the parameter space. For long deadlines, the symmetric equilibrium of an all-pay auction has the same probability measure as an equilibrium of the contest. For short deadlines, both players use symmetric cutoff strategies in the unique equilibrium. In addition, there is an intermediate region in which neither of these two types of equilibria exist. See Fig. 1 for an illustration.

We now compare the different equilibria. For this purpose, we first introduce the concept of a binding deadline.

Definition 2. Consider an equilibrium $\left(\tau^{1}, \tau^{2}\right)$ for a deadline $T$, i.e., $\tau_{1} \leq T, \tau_{2} \leq T$. The deadline $T$ is binding in the equilibrium $\left(\tau^{1}, \tau^{2}\right)$ if there is a deadline $T^{\prime}>T$, a player $i$, and a strategy $\tilde{\tau}^{i} \leq T^{\prime}$, such that

$\pi_{i}\left(\tilde{\tau}^{i}, \tau^{j}\right)>\pi_{i}\left(\tau^{i}, \tau^{j}\right)$. 
Intuitively, if a deadline is binding, a player could increase her profits if she was allowed to unilaterally continue playing after the deadline.

Proposition 7. For any all-pay auction equilibrium, the deadline is not binding. For any other equilibrium, the deadline is binding.

Proof. From the previous proofs, we know that if the expectation of the infinitesimal generator of the payoff process is zero for all $X_{t} \leq \bar{x}-1$ and negative for $X_{t}=\bar{x}$, we have an all-pay auction equilibrium. In this equilibrium, no player can improve his payoff with a longer deadline by the proof of Proposition 2 .

On the other hand, consider an equilibrium in which the expectation of the infinitesimal generator differs from 0 for some $X_{t} \leq$ $\bar{x}-1$. Then, the expectation is necessarily positive at some point $x \leq \bar{x}-1$, since a player would be better off to stop at 0 otherwise. In this case, for any longer deadline $T^{\prime}>T$, the player would be strictly better off using the following strategy: play the equilibrium strategy $\tau$ until the deadline $T$ and continue at $T$ only if $X_{T}=x$ until one additional success occurs. Hence, the deadline is binding.

Hence, the all-pay auction equilibrium is qualitatively different from any other equilibrium in the contest. Even if a player is unilaterally allowed to continue after the deadline, he would have no incentive to do so.

Let us now discuss two examples which show that, in general, there is no monotonic relationship between the size of the deadline and the expected equilibrium effort. Intuitively, a shorter deadline yields two opposing effects. On the one hand, effort might decline because players can only compete up to the deadline. On the other hand, the shorter deadline might make competition before the deadline more fierce. The following examples show that the second indirect effect can offset the first direct effect, but the reverse is also possible.

The expected bid in the symmetric all-pay auction equilibrium equals

$$
\begin{aligned}
\mathbb{E}\left(x^{i}\right) & =\left(1-\frac{\bar{x}}{\hat{p}}\right) \sum_{x=0}^{\bar{x} / 2} 2 x+\left(\frac{1}{\hat{p}}(2+\bar{x})-1\right) \sum_{x=0}^{\bar{x} / 2-1} 2 x+1 \\
& =\left(1-\frac{\bar{x}}{\hat{p}}\right) \frac{\bar{x}}{2}\left(\frac{\bar{x}}{2}+1\right)+\left(\frac{1}{\hat{p}}(2+\bar{x})-1\right) \frac{\bar{x}^{2}}{4}=\frac{\bar{x}}{2}
\end{aligned}
$$

Example 1. Consider the parameters $c=0.255, p=1$, and $\lambda=1$ (as in the proof of Proposition 6). We obtain the expected bid $\mathbb{E}(x)=1$ for the symmetric all-pay auction equilibrium. Players bid up to $\bar{x}=2$ according to $f(0)=f(2)=0.49$ and $f(1)=$ 0.02 . According to Proposition 2 , a lower bound for the deadline for such an equilibrium is $T \geq-\log (0.49)-\log \left(\frac{0.02}{0.51}\right) \approx 3.95$. Expected effort is $\mathbb{E}(x) \approx 1.11$ in the cutoff equilibrium with the longest deadline $T=-W_{-1}\left(-0.51 e^{-1}\right)-1 \approx 1.65$. Players use a cutoff strategy with cutoff 3 in this equilibrium. Thus, expected effort in equilibrium can be higher for a shorter deadline.

Example 2. Consider the parameters $c=0.2, p=1$, and $\lambda=1$. We obtain the expected bid $\mathbb{E}(x)=2$ for the symmetric allpay auction equilibrium. Players bid up to $\bar{x}=4$ according to $f(0)=f(1)=f(2)=f(3)=f(4)=0.2$. We obtain the bound $T \geq-\log (0.2)-\log \left(\frac{0.2}{0.8}\right)-\log \left(\frac{0.2}{0.6}\right)-\log \left(\frac{0.2}{0.4}\right) \approx 4.787$ for the deadline. In the cutoff equilibrium with the longest deadline $T=-W_{-1}\left(-0.4 e^{-1}\right)-1 \approx 2.02$, the expected effort is $\mathbb{E}(x) \approx$ 1.77. Players use a cutoff strategy with cutoff 3 in this equilibrium. Thus, expected effort can be higher for a longer deadline than in the cutoff equilibrium with the longest deadline.
Since we do not have a complete characterization of equilibria for intermediate and long deadlines, we cannot derive conditions about the deadline which maximizes expected effort. Hence, a full characterization of equilibria for all deadlines - which seems technically difficult to obtain - would be an important next step to address the important design question of choosing the optimal deadline.

While the analysis in this paper has been carried out for Poisson processes, the extension to general Markovian arrival processes is straightforward. In this case, the arrival rate $\lambda\left(X_{t}\right)$ need not be constant, but depends on the current level of progress $X_{t}$. The expected time between two arrivals is $\mathbb{E}\left(\tau_{x+1}-\tau_{x}\right)=\frac{1}{\lambda(x)}$. Hence, the symmetric equilibrium distribution of an all-pay auction with the non-linear cost function

$\tilde{c}(x)=c \mathbb{E}\left(\tau_{x}\right)=c \sum_{z=0}^{x-1} \mathbb{E}\left(\tau_{z+1}-\tau_{z}\right)=\sum_{z=0}^{x-1} \frac{c}{\lambda(z)}$

is an equilibrium distribution for the contest with a sufficiently long deadline. The equilibrium distribution $f$ satisfies an indifference equation analogue to Eq. (4)

$0=\frac{p \lambda(x)}{2}(f(x+1)-f(x))-c$.

The proof for Lemma 1 does not depend on the constant arrival rate and a distribution $F$ can be induced before time $T$ if

$T \geq-\sum_{x=0}^{\tilde{x}-1} \frac{1}{\lambda(x)} \log \left(\frac{f(x)}{1-F(x-1)}\right)$.

Similarly, the results for short deadlines can be extended to general Markovian arrival processes. As the final value $X_{T}$ of the process is in general no longer Poisson distributed, however, it is difficult to obtain a closed-form characterization of the cutoff equilibria similar to Lemma 2.

While the extension to general Markovian arrival processes mainly complicates the notation and prohibits closed-form solutions, the extension to semi-Markov processes fundamentally changes the structure of the contest. For a general semi-Markov process, the time between two arrivals is not exponentially distributed, but can follow an arbitrary distribution $G_{x}: \mathbb{R}_{+} \rightarrow[0,1]$, i.e.,

$\tau_{x+1}-\tau_{x} \sim G_{x}$.

As a consequence, the arrival rate need not be constant over time. Thus, the expected change of the payoff process at a value $X_{t}=x$ changes over time. An indifference argument for all points in time as the one in the present paper is not possible anymore. Therefore, we conjecture that equilibria for semi-Markov processes have a different structure than those for Poisson processes.

Throughout this paper, we have focused on the two-player case. For more than two players, there exists no equilibrium characterization for the discrete all-pay auction in the literature, even if symmetry is imposed. The indifference conditions are difficult to handle since each of them contains case distinctions depending on the number of rivals $(1,2, \ldots$, or $N-1)$ who might submit the same bid. Hence, the difference equation cannot be solved in a straightforward way. However, by standard arguments, the equilibrium distribution in any symmetric equilibrium of the all-pay auction needs to have a connected support. Hence, for a sufficiently long deadline, the symmetric equilibrium distribution of the allpay auction is an equilibrium distribution in the corresponding $\mathrm{N}$ player contest. But since there is no analytical solution, no explicit time bound can be derived. For short deadlines, the result from Proposition 4 can be extended to $N$ players, but a characterization 
of the region similar to Proposition 5 is tedious due to the possibility of ties.

\section{Conclusion}

In this paper, we have analyzed the equilibrium structure induced by different deadlines for a contest model in which each player's decision problem is dynamic. For long deadlines, the symmetric equilibrium of an all-pay auction corresponds to an equilibrium of the contest. For short deadlines, equilibrium strategies are cutoff strategies. Intuitively, a shorter deadline reduces the amount of time in which participants can compete, but it increases the intensity of competition. Thus, the contest designer might generate higher efforts with a shorter deadline.

The economic message of the paper is twofold. First, the all-pay auction is a suitable model to analyze contests with a sufficiently long deadline. On the other hand, applying the all-pay reasoning requires some care since a contest designer who wants to maximize expected efforts might be better off with a shorter deadline. We provide a partial characterization for this case.

In the all-pay auction setting, several papers (e.g., Che and Gale, 1998, Dechenaux et al., 2006, and Szech, 2012) analyze bidding caps, i.e., players must not bid above a certain value. For a binding bidding cap, players compensate for the restriction by increasing their probability of bidding at the bidding cap. The contest length $T$, however, restricts the set of stopping times which, in turn, restricts the set of probability distributions. Contrary to a binding bidding cap, the contest length places no restriction on the maximal value at which a player can stop with positive probability.

\section{Acknowledgments}

We are grateful to Paul Heidhues, Martin Hellwig, and Tymon Tatur for their enduring support of this work. Furthermore, we want to thank Matthias Dahm, Alia Gizatulina, Atsushi Kajii, Christian Kellner, Sven Rady, Thomas Rieck, Anja Schöttner, three anonymous referees, and the participants of the micro seminar at Humboldt University Berlin for helpful discussions and remarks. Financial support from the German Research Foundation (DFG) through the Bonn Graduate School of Economics, SFB/TR 15, and GRK 629 is gratefully acknowledged.

\section{Appendix}

Proof of Lemma 1. Denote the first time the process reaches the value $x$ by $s_{x}=\inf \left\{t \geq 0: X_{t} \geq x\right\}$. Define the stopping time $\tau$ as the first time the process stays longer than

$g(x)= \begin{cases}-\frac{1}{\lambda} \log \left(\frac{f(x)}{1-F(x-1)}\right) & \text { if } F(x-1)<1 \\ 0 & \text { if } F(x-1) \geq 1\end{cases}$

at a value $x$ :

$\tau=\inf \left\{t \geq 0: t-s_{X_{t}} \geq g\left(X_{t}\right)\right\}$.

By definition, $s_{X_{t}} \leq t$ is known by time $t$. In addition, the logarithm is well-defined, as $f(x)>0$ for all $x \leq \tilde{x}$. Hence, $\tau$ is a well-defined stopping time. We prove the claim $\mathbb{P}\left(X_{\tau} \leq x\right)=F(x)$ for $\tau$ defined in Eq. (17) by induction.

Induction basis: For $x=0$, we obtain

$$
\begin{aligned}
\mathbb{P}\left(X_{\tau} \leq 0\right) & =\mathbb{P}\left(X_{\tau}=0\right)=\mathbb{P}\left(s_{1}-s_{0}>g(0)\right) \\
& =e^{-\lambda g(0)}=f(0)=F(0) .
\end{aligned}
$$

Induction step: Assume $\mathbb{P}\left(X_{\tau} \leq x\right)=F(x)$. We need to show that this implies $\mathbb{P}\left(X_{\tau} \leq x+1\right)=\bar{F}(x+1)$. As the Poisson process has independent increments, $s_{x+2}-s_{x+1}$ is exponentially distributed independent of the history. Hence,

$$
\begin{aligned}
\mathbb{P}\left(X_{\tau}=x+1\right)= & \mathbb{P}\left(X_{\tau} \geq x+1\right) \mathbb{P}\left(X_{\tau}=x+1 \mid X_{\tau} \geq x+1\right) \\
= & \left(1-\mathbb{P}\left(X_{\tau}<x+1\right)\right) \\
& \times \mathbb{P}\left(s_{x+2}-s_{x+1}>g(x+1)\right) \\
= & (1-F(x)) e^{-\lambda g(x+1)} .
\end{aligned}
$$

Inserting $g(x+1)$ from Eq. (16) yields $\mathbb{P}\left(X_{\tau}=x+1\right)=f(x+1)$. In combination with $\mathbb{P}\left(X_{\tau} \leq x\right)=F(x)$, we obtain the induction step $\mathbb{P}\left(X_{\tau} \leq x+1\right)=F(x+1)$.

The stopping time $\tau$ is bounded from above by the sum of the longest possible times spend at each value $x$ before a player stops. Thus,

$\tau \leq-\frac{1}{\lambda} \sum_{x=0}^{\tilde{x}-1} \log \left(\frac{f(x)}{1-F(x-1)}\right)=\bar{T}$.

Hence, for $T \geq \bar{T}$, we have constructed a strategy which induces $F(x)$.

Proof of Proposition 3. Step 1: Any distribution can be induced by a stopping time.

As in the proof of Lemma 1, denote the first time the process reaches the value $x$ by $s_{x}=\inf \left\{t \geq 0: X_{t}=x\right\}$. Define the stopping time $\tau$ as the first time the process stays longer than

$g(x)= \begin{cases}-\frac{1}{\lambda} \log \left(\frac{f(x)}{1-F(x-1)}\right) & \text { if } f(x)>0 \text { and } F(x-1)<1 \\ \infty & \text { if } f(x)=0 \\ 0 & \text { if } F(x-1) \geq 1\end{cases}$

at a level $x$, i.e.,

$\tau=\inf \left\{t \geq 0: t-s_{X_{t}} \geq g\left(X_{t}\right)\right\}$.

The induction proof is exactly the same as in the proof of Lemma 1. Note that $\mathbb{P}\left(s_{x+2}-s_{x+1}<\infty\right)=1$. Hence, any distribution can be induced for the deadline $T=\infty$.

Step 2: The all-pay auction is strategically equivalent to the contest game for $T=\infty$.

Denote the total expected cost of a bidding distribution $F(x)$ in the all-pay auction by TC ${ }^{\mathrm{APA}}$ and the total expected cost of inducing the distribution $F(x)$ in the contest by $T C^{\mathrm{CON}}$. We obtain

$T C^{\mathrm{APA}}=E(x)=\sum_{x=0}^{\bar{x}} f(x) x=\frac{\bar{x}}{2}$

and

$T C^{\mathrm{CON}}=c E\left(X_{\tau}\right)=c \frac{E(x)}{\lambda}=\frac{c}{\lambda} \sum_{x=0}^{\bar{x}} f(x) x=\frac{c}{\lambda} \frac{\bar{x}}{2}$.

Thus, for $\frac{\lambda p}{c}=\hat{p}$, the cost-price ratio is the same in both games. By step 1, the set of feasible distributions is also the same. Hence, both games are strategically equivalent. Thus, they have the same set of Nash equilibria.

Proof of Lemma 2. We first prove by induction that the inequalities (6) for $x \in\{0,1, \ldots, \bar{x}-2\}$ ensure no player stops at any $x<\bar{x}-1$ in any equilibrium. In the end of this proof, we show that it suffices to verify inequalities (6) for $x \in\{0, \bar{x}-2\}$.

Step 1: A player $i$ strictly prefers to continue at 0 for an infinitesimally small amount of time if

$\left(\mathcal{L} \Pi^{i}\right)(0)=p \lambda \frac{f^{j}(0)+f^{j}(1)}{2}-c>0$ 
The probability that player $j$ stops on 0 or 1 until the deadline $T$ is minimal if the player chooses to continue at these points. Thus, we get

$f^{j}(0)+f^{j}(1) \geq e^{-\lambda T}(1+\lambda T)$.

Combining Eq. (19), Eq. (20), and symmetry of the optimization problems, we obtain that both players strictly prefer to continue at 0 if Eq. (6) is satisfied for $x=0$.

Step 2: Assume that no player ever stops before the deadline $T$ at a value below $x$ with $0<x \leq \bar{x}-2$. In this case, a player $i$ strictly prefers to continue at $x$ for an infinitesimally small amount of time if

$\left(\mathscr{L} \Pi^{i}\right)(x)=p \lambda \frac{f^{j}(x)+f^{j}(x+1)}{2}-c>0$.

Again, the probability that player $i$ stops at $x$ or $x+1$ given that the process reaches $x$ until time $T$ is minimized if the player never stops on $x$ or $x+1$. Given the assumption that no player stops at a value below $x$ before time $T$, we obtain

$f^{j}(x)+f^{j}(x+1) \geq e^{-\lambda T}\left(\frac{(\lambda T)^{x}}{x !}+\frac{(\lambda T)^{x+1}}{(x+1) !}\right)$.

Again, combining Eq. (21), Eq. (22), and symmetry of the optimization problems, we obtain that both players strictly prefer to continue at $x$ if Eq. (6) is satisfied for $x$. This completes the induction.

In the next step, we show that it is optimal to stop at $\bar{x}$. The strategy of the other player that yields the highest incentive to continue at $\bar{x}$ is the cutoff strategy with cutoff $\bar{x}$, because this strategy maximizes the mass on $\bar{x}$ and $\bar{x}+1$. Even in this case, however, (8) ensures that it is optimal to stop at $\bar{x}$. Then, again using the same argument, it is optimal to continue at $\bar{x}-1$ in any equilibrium if (7) holds.

It remains to show that if (6) holds for $x \in\{0, \bar{x}-2\}$, then it also holds for $x \in\{1,2, \ldots, \bar{x}-3\}$. As no player stops below $\bar{x}$ before time $T$, the equilibrium distribution of both players coincides with a Poisson distribution for $x \leq \bar{x}-1$. Since Poisson distributions are single-peaked, we have either

$f(0)+f(1)<f(x)+f(x+1) \quad \forall x \in\{1, \ldots, \bar{x}-3\}$

or

$f(\bar{x}-2)+f(\bar{x}-1)<f(x)+f(x+1)$

$\forall x \in\{1, \ldots, \bar{x}-3\}$.

Since $f(x)+f(x+1)$ is exactly the left-hand side of $(6)$ for any $x \in\{0,1, \ldots, \bar{x}-2\}$ and the right-hand side of $(6)$ is constant, it suffices to verify (6) for $x \in\{0, \bar{x}-2\}$, because the left-hand side is minimal for one of these values by (23) and (24).

Proof of Lemma 3. For every $x \geq \hat{x}$, using the definition of $\hat{x}$,

$\left(\mathcal{L} \Pi^{i}\right)(x) \leq \frac{p \lambda}{2}\left(1-\sum_{y=0}^{\hat{x}-1} \frac{e^{-\lambda T}}{y !}(\lambda T)^{y}\right)-c<0$.

Thus, $\bar{x} \leq \hat{x}$. On the other hand, for every cutoff equilibrium with $\bar{x}<\hat{x}$

$\left(\mathcal{L} \Pi^{i}\right)(\bar{x})=\frac{p \lambda}{2}\left(1-\sum_{y=0}^{\bar{x}-1} \frac{e^{-\lambda T}}{y !}(\lambda T)^{y}\right)-c>0$.

Thus, $\bar{x} \geq \hat{x}$. In combination with $\bar{x} \leq \hat{x}$, in any equilibrium, we have $\bar{x}=\hat{x}$.

Moreover, condition (8) is exactly equivalent to the way we defined $\hat{x}$ and thus automatically satisfied. If condition (7) was to hold with the inverse inequality, the definition of $\hat{x}$ would imply $\hat{x} \leq \bar{x}-1$, a contradiction to $\bar{x}=\hat{x}$. Therefore, (7) also follows directly from the definition of $\hat{x}$.

Proof of Proposition 6. Consider the following parameter setting: $c=0.255, p=1, \lambda=1$. We first show that no cutoff equilibrium and no all-pay auction equilibrium exists for

$$
\begin{aligned}
1.65 & \approx-W_{-1}\left(-0.51 e^{-1}\right)-1<T \\
& <-\log (0.49)-\log \left(\frac{-0.02}{0.49 \log (0.49)}\right) \approx 3.57 .
\end{aligned}
$$

In the second part of this proof, we give a derivation of an equilibrium for $T=1.7$.

In the all-pay auction equilibrium, we have $f(0)=f(2)=$ $0.49, f(1)=0.02$. The quickest stopping time which induces the all-pay auction equilibrium distribution is to stop at $X_{t}=0$ if $t \geq$ $-\log (0.49)$, to stop at $X_{t}=1$ if $t \geq-\log (0.49)-\log \left(\frac{-0.02}{0.49 \log (0.49)}\right)$, and to stop immediately at $X_{t} \geq 2$.

By Propositions 4 and 5, there exists a symmetric cutoff equilibrium with cutoff 1 for $T<-\log (0.49)$ and a symmetric cutoff equilibrium with cutoff 2 if $-\log (0.49)<T<-W_{-1}\left(-0.51 e^{-1}\right)$ -1 . For $T>-W_{-1}\left(-0.51 e^{-1}\right)-1>-\log (0.49)$, no cutoff equilibrium exists according to Proposition 5 . In the cutoff equilibrium with the longest deadline $\left(T=-W_{-1}\left(-0.51 e^{-1}\right)-1\right)$, we obtain $f(0) \approx 0.1927, f(1) \approx 0.3173, f(2) \approx 0.49$.

We now derive a symmetric equilibrium for $T=1.7$. It consists of a cutoff $t_{0}<1.7$ at which each player stops if no success has occurred. If a success occurs for $t<t_{0}$, each player continues until he either has two successes or time $T=1.7$ is reached. We construct the time $t_{0}$ such that a player is indifferent between the optimal continuation strategy and stopping at $X_{t_{0}}=0$. For this purpose, define the continuation payoff at time $t_{0}$ at value 1 by $v\left(1, t_{0}\right)$. It equals the expected gain from an additional success which occurs with probability $1-e^{-\left(1.7-t_{0}\right)}$ minus the expected additional costs,

$$
\begin{aligned}
v\left(1, t_{0}\right) & =\left(1-e^{-\left(1.7-t_{0}\right)}\right) \frac{f(1)+f(2)}{2}-c \mathbb{E}\left[\tau-t_{0} \mid X_{t_{0}}=1\right] \\
& =\left(1-e^{-\left(1.7-t_{0}\right)}\right) \frac{\left(1-e^{-t_{0}}\right)}{2}-c \mathbb{E}\left[\tau-t_{0} \mid X_{t_{0}}=1\right]
\end{aligned}
$$

where $\tau=\min \left\{\inf \left\{t \geq 0: X_{t}=2\right\}, 1.7\right\}$. Note that the expected stopping time is the sum of the expected time for one additional success and the time to the deadline, $1.7-t_{0}$, weighted with the probability that no success occurs:

$$
\begin{aligned}
\mathbb{E}\left[\tau-t_{0} \mid X_{t_{0}}=1\right] & =\int_{0}^{1.7-t_{0}} e^{-t} t d t+\left(1.7-t_{0}\right) e^{-\left(1.7-t_{0}\right)} \\
& =1-e^{-\left(1.7-t_{0}\right)} .
\end{aligned}
$$

Let us now define the point $t_{0}$. At $t_{0}$, a player is indifferent between stopping immediately and continuing with the optimal strategy, i.e., continue if a success occurs at this instant and stop otherwise. Hence, expected infinitesimal gains - direct gains from an additional success plus indirect gains from the continuation payoff at 1 - match expected losses, i.e.,

$\frac{f(0)+f(1)}{2}+v\left(1, t_{0}\right)=0.255$.

By definition of the strategy, $f(0)$ equals the probability that no success occurs until time $t_{0}$ and $f(1)$ is the probability that exactly one success occurs until time $T=1.7$ conditional on at least one success until time $t_{0}$. Thus, the indifference equation becomes

$$
\begin{gathered}
\frac{e^{-t_{0}}+e^{-1.7} t_{0}}{2}+\frac{\left(1-e^{-t_{0}}\right)\left(1-e^{-\left(1.7-t_{0}\right)}\right)}{2} \\
-0.255\left(1-e^{-\left(1.7-t_{0}\right)}\right)=0.255 .
\end{gathered}
$$


We obtain $t_{0} \approx 1.6449$ for $T=1$.7. Plugging in, we obtain $f(0) \approx$ $0.1930, f(1) \approx 0.3005$ and $f(2) \approx 0.5065$. By construction, it is optimal to continue at 0 until time $t_{0}$. Moreover, it is always optimal to continue at 1 , since the expectation of the infinitesimal generator of the payoff process is positive for $x=1$. Similarly, it is always optimal to stop at values above 1 , since the expectation of the infinitesimal generator is negative. Hence, we have constructed an equilibrium for $T=1.7$.

\section{References}

Baye, M., Kovenock, D., de Vries, C., 1994. The solution to the Tullock rent-seeking game when $R>2$ : mixed-strategy equilibria and mean dissipation rates. Public Choice 81, 363-380.

Baye, M., Kovenock, D., de Vries, C., 1996. The all-pay auction with complete information. Economic Theory 8 (2), 291-305.

Bos, O., 2012. Wars of attrition and all-pay auctions with stochastic competition, 48 (2), 83-91.
Che, Y.-K., Gale, I., 1998. Caps on political lobbying. American Economic Review 88 (3), 643-651.

Cohen, C., Sela, A., 2007. Contests with ties. The B.E. Journal of Theoretical Economics 7 (1), 1-16.

Dechenaux, E., Kovenock, D., Lugovskyy, V., 2003. A comment on 'David vs. Goliath: an analysis of asymmetric mixed-strategy games and experimental evidence'. Mimeo.

Dechenaux, E., Kovenock, D., Lugovskyy, V., 2006. Caps on bidding in all-pay auctions: comments on the experiments of A. Rapoport and W. Amaldoss. Journal of Economic Behavior and Organization 61, 276-283.

Hillman, A., Riley, J., 1989. Politically contestable rents and transfers. Economics and Politics 1, 17-39.

Hillman, A., Samet, D., 1987. Dissipation of contestable rents by small numbers of contenders. Public Choice 54, 63-82.

Rogers, L.C.G., Williams, D., 2000. Diffusions, Markov Processes and Martingales Vol. 1. Cambridge University Press.

Seel, C., Strack, P., 2013. Gambling in contests. Journal of Economic Theory 148 (5), 2033-2048.

Siegel, R., 2009. All-pay contests. Econometrica 77 (1), 71-92.

Szech, N., 2012. Tie-breaks and bid-caps in all-pay auctions. Mimeo.

Taylor, C., 1995. Digging for golden carrots: an analysis of research tournaments. American Economic Review 85 (4), 872-890. 